CERN-TH/97-355

hep-ph/9712298

December 1997

\title{
QUARKONIUM POLARIZATION AS A TEST OF NON-RELATIVISTIC EFFECTIVE THEORY
}

\author{
M. BENEKE \\ Theory Division, CERN, CH-1211 Geneva 23 \\ I compare current approaches to quarkonium production with regard to what they \\ tell us about quarkonium polarization. Predictions for $J / \psi$ polarization in hadron- \\ hadron and photon-hadron collisions are summarized.
}

To appear in the Proceedings of the International Europhysics Conference on High Energy Physics, Jerusalem, Israel, 19-26 August 1997 
The production of charmonium involves physics at short and long distances. The short-distance part is given, uncontroversially, by the production cross section for a charm-anticharm-quark pair at small relative velocity $v$ of the two quarks. The process through which the $c \bar{c}$ pair binds into a particular charmonium state is sensitive to long times $\tau \sim 1 /\left(m_{c} v^{2}\right) \sim 1 /(500 \mathrm{MeV})$ and therefore it is non-perturbative. Various descriptions, with more or less contact to QCD, and relying on quite different physical pictures of this process, haye been proposed, and used, over the years. The colour-singlet model $(C S M) \square$ assumes that only those $c \bar{c}$ pairs form $J / \psi$ which are produced in a colour-singlet ${ }^{3} S_{1}$ state already at short distances. The long-distance part is Coulomb-binding, accountable for by the wave-function at the origin. No gluons with energy less than $\mathcal{O}\left(z_{c}\right)$ in the $J / \psi$ rest frame are emitted. The colour-evaporation model (CEM) 1 assumes that soft gluon emission from the $c \bar{c}$ pair is unsuppressed. The colour and spin quantum numbers of the $c \bar{c}$ pair at short distances are irrelevant. The long-distance physics is supposed to be described by a phenomenological parameter $f_{J / \psi}$, the fraction of 'open' $c \bar{c}$ pairs below threshold that bind into $J / \psi$. The non-relativistic $Q C D$ (NRQCD) approach 3 synthesizes elements of both approaches. $J / \psi$ can be produced from $c \bar{c}$ pairs in any colour or angular momentum state at short distances but with probabilities that follow definite scaling rules 4 in $v^{2}$. Soft gluon emission does take place, but the interaction of soft gluons with the heavy quarks is determined by the NRQCD effective Lagrangian. Spin symmetry holds to leading order in $v^{2}$. There is a price to pay for the more detailed description of the long-distance part in NRQCD: It depends on (at least) four (rather than one) non-perturbative parameters, which have to be extracted from experiment. They are $\left\langle\mathcal{O}_{1}^{J / \psi}\left({ }^{3} S_{1}\right)\right\rangle,\left\langle\mathcal{O}_{8}^{J / \psi}\left({ }^{3} S_{8}\right)\right\rangle,\left\langle\mathcal{O}_{8}^{J / \psi}\left({ }^{1} S_{0}\right)\right\rangle,\left\langle\mathcal{O}_{8}^{J / \psi}\left({ }^{3} P_{0}\right)\right\rangle$, where the colour and angular momentum state indicated refers to the $c \bar{c}$ pair at short distances. The precise definition of these matrix elements is given in 3 .

The following deals exclusively with polarization phenomena in $J / \psi$ production. We discuss predictions for $J / \psi$ production in hadron-hadron and photon-proton collisions, based on the CSM and the NRQCD approach. The prediction by the CEM is straightforward and universal: Because the model assumes that transitions ${ }^{3} S_{1} \leftrightarrow{ }^{1} S_{0}$ are unsuppressed, we expect that $J / \psi$ is always produced unpolarized. A polarization measurement has various discriminative powers. One can learn to what degree spin-flip transitions are suppressed and thereby check the basic assumption that distinguishes the CEM from the NRQCD approach. Since the octet production matrix elements of NRQCD (see above) lead to a polarization pattern different from the CSM, one can learn about the importance of colour-octet production mechanisms. In particular, production through a ${ }^{1} S_{0}^{(8)}$ state yields unpolarized quarkonium. 
See国国 for the other production channels.

$J / \psi$ production in fixed target hadron-hadron collisions. Polarization measurements exist for $\psi$ and $\psi^{\prime}$ production in pion scattering fixed target experiments 6 . Both experiments observe an essentially flat angular distribution in the decay $\psi \rightarrow \mu^{+} \mu^{-}\left(\psi=J / \psi, \psi^{\prime}\right)$,

$$
\frac{d \sigma}{d \cos \theta} \propto 1+\lambda \cos ^{2} \theta
$$

where the angle $\theta$ is defined as the angle between the three-momentum vector of the positively charged muon and the beam axis in the rest frame of the quarkonium. The observed values for $\lambda$ are $0.02 \pm 0.14$ for $\psi^{\prime}$, measured at $\sqrt{s}=21.8 \mathrm{GeV}$ in the region $x_{F}>0.25$ and $0.028 \pm 0.04$ for $J / \psi$ measured at $\sqrt{s}=15.3 \mathrm{GeV}$ in the region $x_{F}>0$.

The colour-singlet contribution alone yields $\lambda \approx 0.25$ for the direct $S$-wave production cross section 9 . However, the total cross section is largely due to colour-octet production. The polarization in the colour-octet channels has been considered in (see also 10$)$. If $\left\langle\mathcal{O}_{8}^{\psi^{\prime}}\left({ }^{1} S_{0}\right)\right\rangle$ and $\left\langle\mathcal{O}_{8}^{\psi^{\prime}}\left({ }^{3} P_{0}\right)\right\rangle$ are constrained to be positive, $0.15<\lambda<0.44$ is obtained for $\psi^{\prime}$ production at $\sqrt{s}=21.8 \mathrm{GeV}$. The lower bound is obtained if production through a $c \bar{c}\left[{ }^{1} S_{0}^{(8)}\right]$ intermediate state dominates. The analysis of $J / \psi$ polarization is complicated by indirect $J / \psi$ production through $\chi_{c}$ decays, which are not separated in the measurement above. In Fig. 1 the polar angle parameter $\lambda$ is plotted as a function of $r_{L}$, the longitudinal polarization fraction of indirectly produced $J / \psi$ (i.e. $r_{L}=1 / 3$, if $\chi_{c}$ feed-down gives unpolarized $\left.J / \psi\right) \cdot r_{L}$ is difficult to obtain theoretically as $\chi_{c 1}$ and perhaps even $\chi_{c 2}$ is dominantly produced through colour-octet states, whose polarization yield is described by too many phenomenological parameters to be predictable. The wide band in Fig. 1 is obtained by saturating the direct $J / \psi$ production cross section by either $\left\langle\mathcal{O}_{8}^{J / \psi}\left({ }^{1} S_{0}\right)\right\rangle$ (lower curve) or $\left\langle\mathcal{O}_{8}^{J / \psi}\left({ }^{3} P_{0}\right)\right\rangle$ (upper curve). If the indirectly produced $J / \psi$ are unpolarized, one would again have to assume that $\left\langle\mathcal{O}_{8}^{J / \psi}\left({ }^{1} S_{0}\right)\right\rangle \gg\left\langle\mathcal{O}_{8}^{J / \psi}\left({ }^{3} P_{0}\right)\right\rangle$ in order to reproduce the data (horizontal band in Fig. 1). A measurement of $r_{L}$ could clarify the situation.

Since the total cross section is dominated by $J / \psi$ production at small transverse momentum, non-factorizable final state interactions may be significant (though formally suppressed) and invalidate the predictions based on the CSM or NRQCD.

$J / \psi$ polarization at the Tevatron. At transverse momentum $p_{t} \gg 2 m_{c}$ $J / \psi$ production in hadron-hadron collisions is now regarded as a gluon frag- 


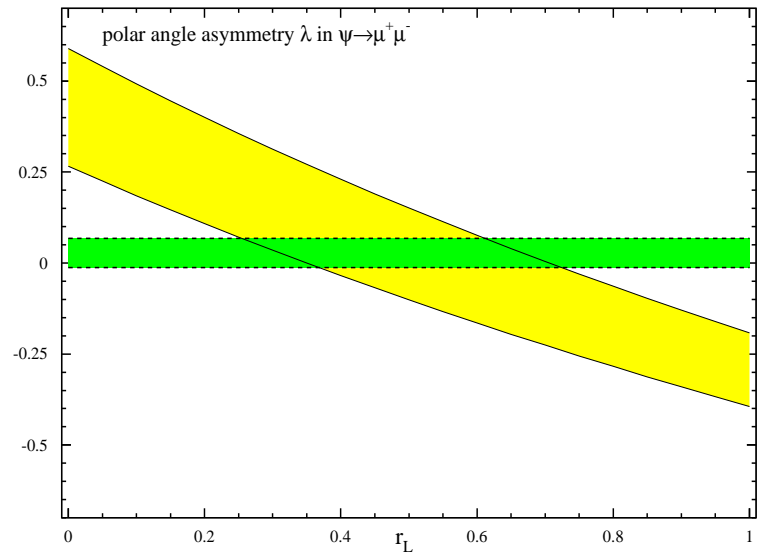

Figure 1: Polar angle asymmetry for $J / \psi$ production in pion-nucleus collisions at $\sqrt{s}=$ $15 \mathrm{GeV}$ as a function of the longitudinal polarization fraction of indirect $J / \psi$ from radiative feed-down. The horizontal band shows the measurement of $\lambda$.

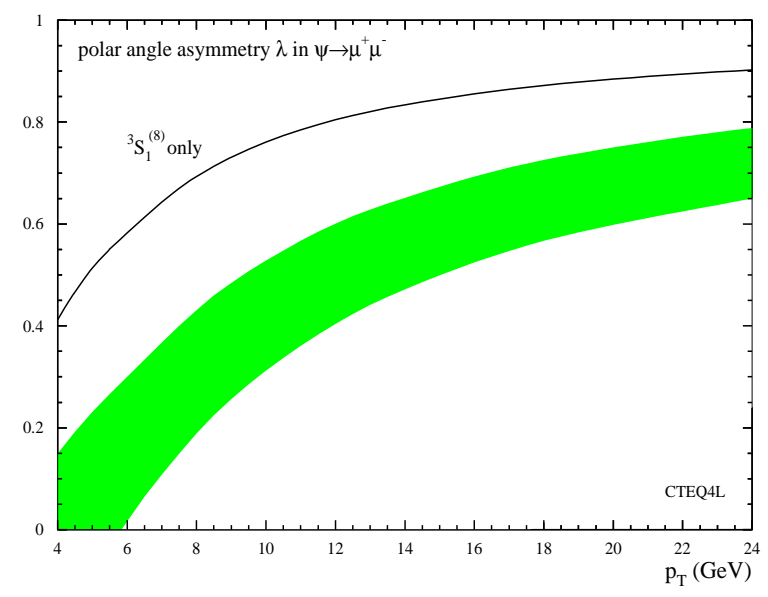

Figure 2: $\lambda$ as a function of $p_{t}$ in $p+\bar{p} \rightarrow J / \psi+X$ at the Tevatron cms energy $\sqrt{s}=1.8$ TeV. From 13 . 
mentation process $g \rightarrow c \bar{c}\left[{ }^{3} S_{1}^{(8)}\right] \rightarrow J / \psi+X$ 目. Since the fragmenting gluon is nearly on-shell, this implies transversely polarized $J / \psi$ as $p_{t} \rightarrow \infty \mathbb{1}$ up to spin-symmetry breaking corrections of order $v^{4}$. At finite $p_{t}$ longitudinally polarized $J / f$ can be produced, if a hard gluon is radiated in the fragmentation process 5 or the fragmentation approximation is relaxed 13,43 . The non-fragmentation terms turn out to be particularly important. The predicted polar angle asymmetry $\lambda$ is shown in Fig. 2. At $p_{t} \sim 5 \mathrm{GeV}$ no trace of transverse polarization remains. As $p_{t}$ increases, the angular distribution becomes rapidly more anisotropic. The observation of this pattern, even qualitatively, would already constitute strong support for the gluon fragmentation mechanism and the relevance of spin symmetry in quarkonium production. The polarization measurement will therefore rule out either the CEM or the applicability of NRQCD velocity power counting at the charmonium scale. Because of this, this measurement is probably the single most important one that can be done in the near future.

$J / \psi$ photo-production at HERA. This production process deserves special attention in the context of NRQCD (and the CEM), since the colour-octet contributions to the energy distribution of inelastically produced $J / \psi$ seem to be too large close to the point $z=1$ of maximal energy transfer 15. Since the NRQCD velocity expansion is not valid in this endpoint region 16 , one would like to infer the relevance of the colour-octet contribution from the data themselves. Polar and azimuthal $J / \psi$ decay angular distributions may provide a clue to the answer to this problem, since the distributions are predicted to be quite different in the CSM and in the NRQCD approach 17 . [Recall that all angular distributions are isotropic in the $J / \psi$ rest frame in the CEM.] The azimuthal dependence, characterized by two additional angular parameters $\mu$ and $\nu$, is particularly instructive, as a function of both energy fraction $z$ or transverse momentum as shown in Fig. 3. The shaded band reflects the variation that follows if either one of $\left\langle\mathcal{O}_{8}^{J / \psi}\left({ }^{1} S_{0}\right)\right\rangle,\left\langle\mathcal{O}_{8}^{J / \psi}\left({ }^{3} P_{0}\right)\right\rangle$ is set to zero, while the other saturates the sum which is constrained by the total production rate (in other production processes, primarily hadron-hadron collisions as discussed above). A measurement of angular distributions in inelastic $J / \psi$ production comparable to the measurement of polarization in diffractive $J / \psi$ production at HERA 18 could resolve the controversy whether the measured energy distribution is described by the CSM alone and/or is in conflict with the size of colour-octet contributions suggested by the NRQCD approach.

In the framework of NRQCD predictions of $J / \psi$ polarization have also been obtained for $B \rightarrow J / \psi+X €$, direct $J / \psi$ production in $Z^{0}$ decay 20 and 

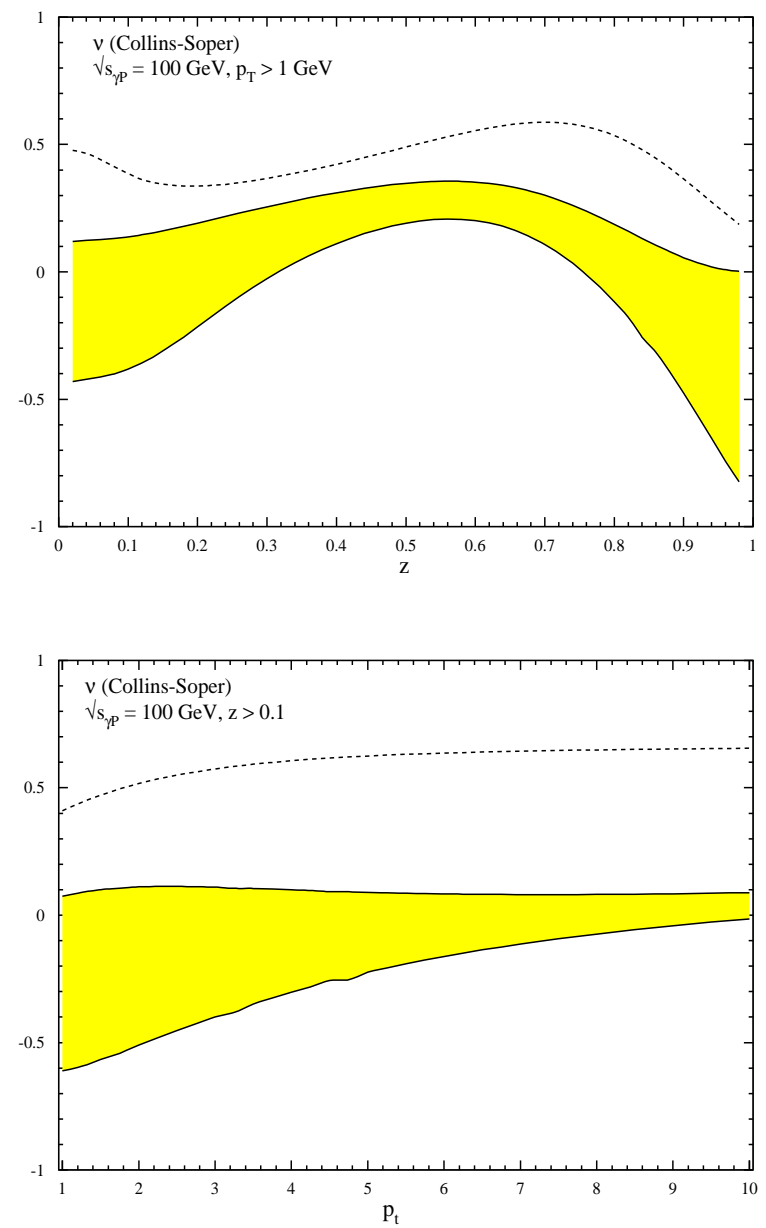

Figure 3: Azimuthal angle parameter $\nu$ in the Collins-Soper frame for $J / \psi$ energy and transverse momentum distribprions in $J / \psi$ photo-production at a typical HERA energy. From 4 where more details can be found. 
lepto-production of $J / \psi 21$. Because of lack of space, the reader is referred to the original papers.

Acknowledgements. I would like to thank M. Krämer, I.Z. Rothstein and M. Vänttinen for their collaboration on this topic.

1. For a review, see G.A. Schuler, CERN-TH-7170-94 hep-ph/9403387, and references therein.

2. H. Fritzsch, Phys. Lett. B67, 217 (1977); F. Halzen, Phys. Lett. B69, 105 (1977).

3. G.T. Bodwin, E. Braaten and G.P. Lepage, Phys. Rev. D51, 1125 (1995) [Erratum: ibid. D55, 5853 (1997)].

4. G.P. Lepage et al., Phys. Rev. D46, 4052 (1992).

5. M. Beneke and I.Z. Rothstein, Phys. Lett. B372, 157 (1996) [Erratum: ibid. B389, 789 (1996)].

6. M. Beneke and I.Z. Rothstein, Phys. Rev. D54, 2005 (1996) [Erratum: ibid. D54, 7082 (1996)].

7. E. Braaten and Y.Q. Chen, Phys. Rev. D54, 3216 (1996).

8. J.G. Heinrich et al., Phys. Rev. D44, 1909 (1991); C. Akerlof et al., Phys. Rev. D48, 5067 (1993).

9. M. Vänttinen et al., Phys. Rev. D51, 3332 (1995).

10. W.-K. Tang and M. Vänttinen, Phys. Rev. D54, 4349 (1996).

11. E. Braaten and S. Fleming, Phys. Rev. Lett. 74, 3327 (1995).

12. P. Cho and M.B. Wise, Phys. Lett. B346, 129 (1995).

13. M. Beneke and M. Krämer, Phys. Rev. D55, 5269 (1997).

14. A.K. Leibovich, Phys. Rev. D56, 4412 (1997).

15. M. Cacciari and M. Krämer, Phys. Rev. Lett. 76, 4128 (1996); P. Ko, J. Lee and H.S. Song, Phys. Rev. D54, 4312 (1996).

16. M. Beneke, I.Z. Rothstein and M.B. Wise, Phys. Lett. B408, 373 (1997).

17. M. Beneke, M. Krämer and M. Vänttinen, hep-ph/9709376.

18. H1 Collaboration, S. Aid et al., Nucl. Phys. B472, 3 (1996) and the update Paper pa02-085, submitted to the 28th International Conference on High Energy Physics, ICHEP'96, Warsaw, Poland, July 1996; ZEUS Collaboration, J. Breitweg et al., Z. Phys. C75, 215 (1997).

19. S. Fleming et al., Phys. Rev. D55, 4098 (1997).

20. S. Baek et al., Phys. Rev. D55, 6839 (1997).

21. S. Fleming and T. Mehen, JHU-TIPAC-96022 hep-ph/9707365. 